\title{
The Structure of the Electric Double Layer: Atomistic vs. Continuum Approaches
}

\author{
Sung Sakong, ${ }^{1, *}$ Jun Huang, ${ }^{1,2}$ Michael Eikerling, ${ }^{2}$ and Axel Groß ${ }^{1,3, \dagger}$ \\ ${ }^{1}$ Institute of Theoretical Chemistry, \\ Ulm University, 89069 Ulm, Germany \\ ${ }^{2}$ IEK-13, Forschungszentrum Jülich, \\ Wilhelm-Johnen-Straße, 52425 Jülich, Germany \\ ${ }^{3}$ Helmholtz Institute Ulm (HIU), Electrochemical Energy Storage, 89069 Ulm, Germany
}

\begin{abstract}
The theoretical modeling of the double layer structure at electrode/electrolyte interfaces by current atomistic and continuum approaches is reviewed. We will briefly discuss recent progress in both approaches and present a perspective on how to better describe the electric double layer by exchanging the unique advantages of each method. First-principles atomistic approaches provide detailed insights into the electronic and geometric structure of electrode/electrolyte interfaces. However, they are numerically too demanding to allow a systematic study of the properties of electric double layers for a wide range of electrochemical conditions. Still they can provide valuable input for continuum approaches which due to their numerical efficiency can capture the influence of the electrochemical environment on larger length and time scale. Still, these methods rely on reliable input parameters. Conversely, continuum methods can provide a preselection of interface structures and conditions to be further studied on the atomistic level.

Keywords: Electric double layer, Semiclassical method, Ab initio molecular dynamics simulations, Electrochemical interfaces
\end{abstract}

\footnotetext{
* sung.sakong@uni-ulm.de

$\dagger$ axel.gross@uni-ulm.de
} 


\section{INTRODUCTION}

Electrochemistry is concerned with structures and processes at the interface between an electron conductor, the electrode, and an ion conductor, the electrolyte $[1,2]$. At the interface between a solid electrode and liquid electrolyte, an electric double layer (EDL) is formed by rearranging the electronic charges and ions according to the electrochemical conditions. The EDL exhibits spatiotemporally fluctuating local polarization due to the liquid nature of electrolytes. Because of the large numerical effort associated with the explicit modeling of the atomistic structure, the representative properties of the EDL are usually described by statistically averaged values. One of the most important properties is the electrostatic potential governed by the polarization distribution in the EDL area, created by the electronic and ionic charges. Thus, modern EDL theories focus on identifying and modeling the polarization elements, e.g., the orientation of solvent molecules, ion arrangements, and the redistribution of electrons [3-10].

The traditional classical approaches assume a Boltzmann distribution of the ions in the electrolyte and derive the effective electric potential by solving the Poisson equation. The quality of the methods depends on the aptness and completeness in the description of the polarization elements, which are included in different levels of depths in existing theories. On the one hand, there are continuum approaches rooted in a very long tradition (see, e.g., Ref. [2]), which still are being further improved. An example of the ongoing progress made in this field is a recently developed classical model that attempts to provide a computationally efficient grand-canonical scheme by treating the electrode and electrolyte phases on the same footing [7-9]. On the other hand, there are atomistic approaches which in combination with

density functional theory (DFT) can highly accurately evaluate the charge polarization and the electrostatic potential when a suitable atomic configuration is known. The statistical EDL properties can be sampled along a trajectory using a model configuration by ab initio molecular dynamics (AIMD) simulations [3-6, 10-13].

We will compare the recent progress of the atomistic and semiclassical continuum approaches and present a perspective of how to integrate findings from both methods for advances of the EDL models in the future. 


\section{SEMICLASSICAL DENSITY-POTENTIAL FUNCTIONAL THEORY}

TABLE I. Comparison between AIMD and semiclassical methods. The semiclassical approach combines theories on different scales for the electrode and electrolyte, while AIMD simulations treat all parts on a same level.

\begin{tabular}{lcc}
\hline & AIMD & Semi-classical \\
\hline Scale & Microscopic & Multiscale \\
Ensembles & Canonical $N V T$ & Grand canonical $\mu V T$ \\
Constraints & $N_{e l}$ & $\mu_{e l}, \mathrm{pH}, \cdots$ \\
Sampling time & $<100 \mathrm{ps}$ & Static \\
System size & $<10^{3}$ atoms & $N_{A}$ particles \\
Computer time & $\sim 1 \mathrm{M} \mathrm{CPU}$ hours & Several minutes for $1 \mathrm{D}$ model \\
\hline Statistical average $\langle A\rangle=$ & $\frac{1}{T} \int_{t=0}^{T} A(\{R\}, n ; t) d t$ & $\mathcal{A}$ \\
Electrode potential $U$ & Work function $\Phi(t)$ & Electron chemical potential $\mu_{e l}$ \\
Electric potential $\phi(z)$ & $\int_{A} \phi(r, t) d x d y / A$ & Inner potential $\varphi(z)$ \\
Structural factors & Atomic coordinates $\{R(t)\}$ & Gap $t$, ionic cores $a_{m c}, \cdots$ \\
\hline
\end{tabular}

The semiclassical EDL model by Huang et al. combines an orbital-free quantummechanical description of the electrode and a classical statistical field description of the electrolyte, as sketched in Fig. 1 [7-9]. Presently, the kinetic and exchange-correlation energies of electrons are addressed by the Thomas-Fermi-von Weizsäcker and Dirac-Wigner theories, respectively. Metal cation cores are represented as line positive charges in a onedimensional configuration. A classical density-potential functional theory (DPFT) describes the electrolyte solution considering the asymmetric steric effects and solvent polarization in the electrolyte solution and ion-specific interactions of electrolyte species with the electrode $[9]$.

The grand potential of the whole EDL then includes the electrode, electrolyte, and the interactions between them. A variational analysis of the grand potential yields a grandcanonical picture of the EDL using two Euler-Lagrange equations in terms of the electron density and the electric potential. By avoiding the Kohn-Sham orbital optimization, the 


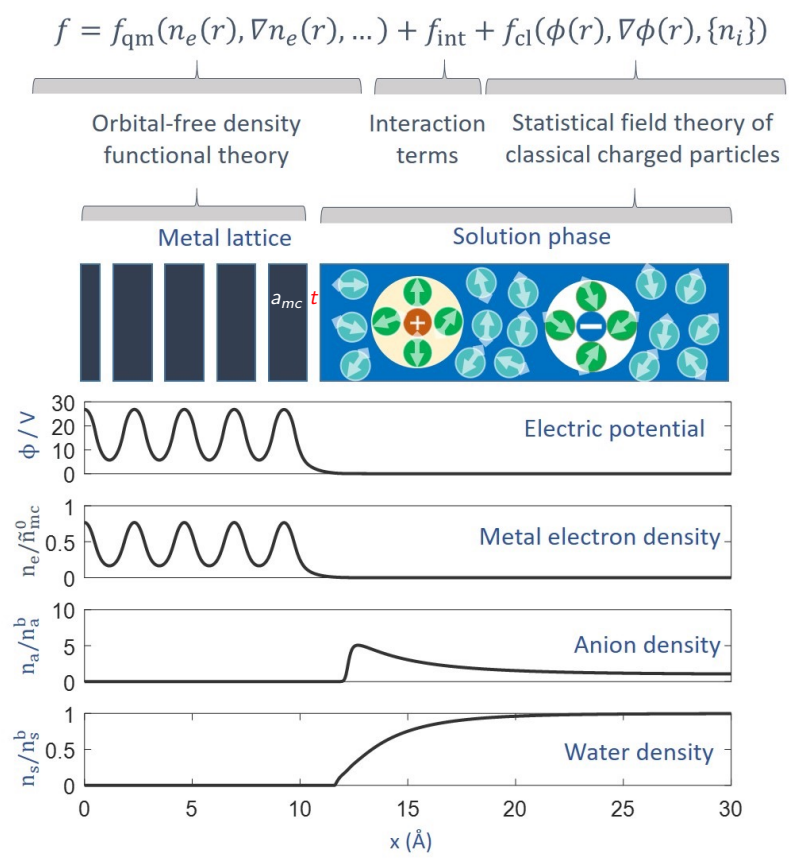

FIG. 1. A hybrid density-potential functional theory for the EDL. The EDL is divided into an inhomogeneous electron gas described by quantum mechanical DFT, and an inhomogeneous Coulombic fluid described by classical statistical field theory. This model is different from the joint DFT model developed by Arias et al. on two aspects $[14,15]$. Firstly, orbital-free DFT is used and the electron density distribution is solved from a Euler-Lagrange equation with negligible computational cost. Therefore, this model is promising to simulate realistic EDLs. Secondly, a hybrid DPFT is developed to describe the electrolyte solution considering asymmetric steric effects, solvent polarization, and ion-specific interactions with the metal. These factors are not considered in the Poisson-Boltzmann theory used in the joint DFT. Subplots show the distributions of the electric potential, the metal electron density normalized to the electron density of the metal cationic cores, the anion density and the water molecule density, both normalized to their bulk values, from the metal phase to the solution phase [8].

computational cost of this approach becomes negligible compared with Kohn-Sham DFTbased methods, such as the joint DFT developed by the Arias group [14, 15].

The semiclassical method provides a computationally efficient description of EDL properties within a grand canonical scheme, i.e., it describes the electrode and the electrolyte solution on equal footings. Figure 1 shows the EDL properties determined under a con- 
stant potential condition, which include the oscillating electron density in the metal lattice, electron spillover from the electrode, accumulation (depletion) of counterions (coions) in the diffuse layer, the field-dependent orientation of solvent molecules, and partial charge transfer, if any, described using an Anderson-Newns type model [8].

However, the orbital-free DFT is a crude approximation [16, 17], and its accuracy in an electrochemical environment is still unknown. The chemical interaction between ions/molecules in solution and the electrode, which is essential for chemisorption and more complicated electrocatalytic phenomena, requires further attention. In addition, the semiclassical model is currently unable to optimize the structure of the EDL. Most importantly, it introduces a structural parameter, the gap between the species (solvent molecules and ions) in the electrolyte and cationic cores in the electrode, which determines all important EDL properties. For example, stronger specific ion adsorption could result in a smaller gap $t$ between the electrode and electrolyte, and it causes a smaller potential of zero charge (pzc) and an elevated double-layer capacitance curve [8].

\section{AIMD SIMULATIONS}

DFT calculations yield the total energy, the electron distribution, and the forces acting on the atoms upon solving the Kohn-Sham equation. The EDL properties can then be statistically determined by molecular dynamics simulations using the ergodic theorem. Kohn-Sham DFT is known to describe metal-metal, metal-adsorbate, and water-water interactions with a high reliability $[11,19]$, but it is computationally more demanding compared to the orbitalfree DFT. A typical electrochemical property that can be determined by AIMD simulations in good agreement with the experiment is the potential of zero charge $[3,4,10,12]$ which can be derived from the work function of an ion-free water film above a metal slab [20] as illustrated in Fig. 2.

However, due to their high computational demand, AIMD simulations in the canonical ensemble can only consider relatively small systems for limited run times typically below 100 ps (see Table I). The representation of electrified interfaces poses a particular challenge. In periodic calculations, the unit cell has to be electrostatically neutral, and any surplus charge will be automatically compensated by a homogeneous charge background. In principle, electrochemical interfaces also have to be charge-neutral, and any excess charge in 

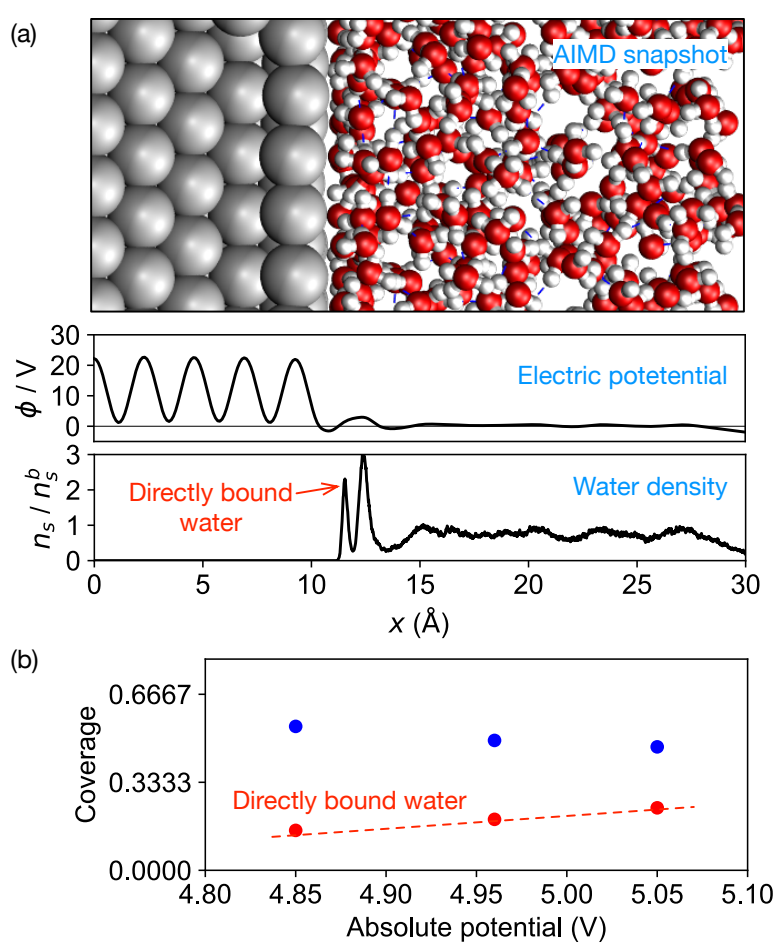

FIG. 2. (a) AIMD simulations of the interface between $\operatorname{Pt}(111)$ and water at the pzc. The unit cell consists of 612 atoms and 2952 electrons. The statistical average has performed over 40 ps by solving Langevin equation at $293 \mathrm{~K}[4,18]$. (b) The coverage of solvating water molecules as a function of the electrode potential [18].

the EDL will be balanced by the corresponding counter charge in the electrode. Within the DFT, there are two approaches to reproduce the compensating polarization. Firstly, an appropriate EDL configuration can be tailored using explicit ions. In practice, by adding a charge-neutral atom of a target ion into the water film, the electrons of the atom are transferred to the Fermi level of the electrode, and the exchange of electrons between the electrode and the atom species creates ion species in the water film [4, 21-23]. Secondly, the compensating polarization can be implicitly modeled by the density difference of positive and negative ions modeled by the Boltzmann distribution. The net polarization distribution is optimized with the electron density by simultaneously solving the Kohn-Sham and the linearized Poisson-Boltzmann equations [14, 24-26]. 

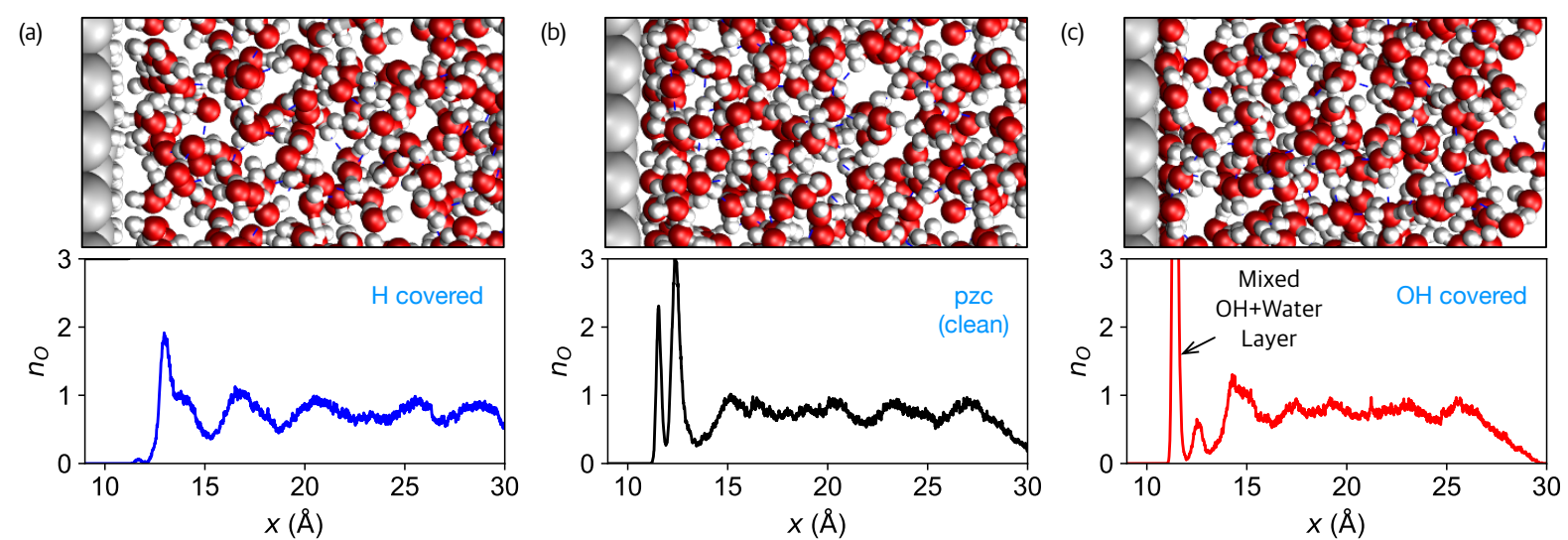

FIG. 3. O atom distribution in the water films on (a) $\mathrm{H}$ covered, (b) clean, and (c) $\mathrm{OH}$ covered Pt(111) electrodes. On H covered electrode, no directly bound water molecules are found, whereas the directly bound water and $\mathrm{OH}$ molecules form a compact solvation layer on $\mathrm{OH}$ covered electrode $[4,18]$.

\section{BRIDGING AIMD AND SEMICLASSICAL METHODS}

After briefly discussing the strengths and weaknesses of both the semiclassical method and the AIMD approaches, we will now address how these two approaches can be interconnected. The semiclassical method models the EDL within a grand canonical scheme, whereas the AIMD simulation yields atomistic details of the interface. The atomic configurations presenting polarization elements identified in AIMD simulations can be an input for the improved parametrization of the semiclassical method. Conversely, the semiclassical method can guide preparing and validating the atomic setup in AIMD simulations.

One of the factors determining the polarization at the interface is the particular water structures occurring along the AIMD trajectories that substantially contribute to the statistical ensemble along the calculated trajectories. As already demonstrated by early simulations employing an ice-like bilayer model on close-packed metal surfaces [27], a purely ionic picture relying only on the orientation of the $\mathrm{O}-\mathrm{H}$ bonds is not sufficient to capture the formation of the interface dipole, especially on transition metals. The charge analysis shows that a considerable electron transfer occurs from the water layer to the metal, contributing to the interface dipole. These findings were confirmed by the analysis of AIMD simulations using several water layers on metal surfaces $[3,4,6]$. In addition to the water 
orientation, they identified a densely packed water solvation layer with a strong electronic charge redistribution contributing to the overall polarization of the interface.

In more detail, Li et al. [10] demonstrated that the strong bonding of the water molecules to the surface (see Fig. 2a) weakens one of the O-H bonds, which supports the instant formation of Hydronium or Zundel configurations in the solvation layer on transition metal surfaces $[4,5,10,12,13]$ and thus contributes to the polarization of the liquid water. As Le et al. showed [5], the weakened $\mathrm{O}-\mathrm{H}$ bonds are associated with a redshift of the $\mathrm{O}-\mathrm{H}$ stretch vibration facilitating the proton motion at the interface through the Grotthuss mechanism, which causes a higher ion activity at the interface than in bulk liquid water. Furthermore, Lan et al. showed that nuclear quantum effects could more enhance the proton activity in the solvation layer [13].

The particular water configurations at the interface critically determine the relative potentials between bulk liquid water to the electrode phases. As demonstrated by recent AIMD simulations, the electric potential in the bulk water potential is placed much closer to the Fermi level when the electrolyte is explicitly modeled by water molecules, compared to an implicit solvent $[28,29]$. The missing configurations associated with an additional dipole formation in the implicit solvent lead to the lack of charge transfer at the interface and subsequently too small interface dipoles. As identified by Li et al. [10], the Helmholtz model can capture the polarization properties of the strongly bound water molecules. The specific water configuration equivalent to the formation of a Helmholtz layer is only stable when the metal-water interaction is strong enough, like on transition metal electrodes. On noble metal electrodes like $\mathrm{Ag}$ and $\mathrm{Au}$, where the metal-water interaction is weak, the formation of such a water configuration is less pronounced. The corresponding semiclassical assessment requires the gap parameter $t[8,30]$, which is related to the distance to the Helmholtz layer. The parameter $t$ also needs to reflect the interaction strength between metal and water and further considerations than the cation spacing.

One of the $\mathrm{OH}$ bonds of most water molecules points toward the electrode on $\mathrm{Pt}(111)$. A delicate polarization change at the interface by populating ion species in the water film leads to a linear relationship between the coverage of directly bound water molecules to the electrode and the work function. As shown in Fig. 2b, the density of the solvation layer is insensitive to the modest variation of the polarization, but there is an exchange between the directly bound water molecules (red dots) and the solvating molecules with pointing the 
$\mathrm{OH}$ toward the electrode (blue dots). The increase of the number of directly bound water molecules is in line with the increase of the interface dipole.

Since the structural change in the solvation layer is a response to the field created by the electrolyte polarization $[14,25,26,31,32]$, it is not trivial to faithfully reproduce this effect in the parametrization of implicit solvent methods through the solvation cavity and local permittivity at the contact [33]. There are advanced approaches to address the solvation structures without relying on the cavity, namely the reference interaction site method (RISM) [34, 35], but the delicate structures of the solvation layer in Fig. 3 are still challenging to be captured within the approach.

The adsorbed species also change the polarization at the interface $[4,10,36-39]$. The $\mathrm{H}$ adsorption blocks the direct adsorption of water molecules and increases the distance between the solvating water molecules and the electrode by around $1 \AA$ at low potentials, as shown in Fig. 3. The increased distance between the water molecules and the surface weakens their interaction. Consequently, the $\mathrm{H}$ adsorbates block the direct adsorption of water molecules on the electrode. When anion species cover the electrode, e.g., $\mathrm{OH}^{-}$[40-42], they alter the solvation layer structure significantly. As recently demonstrated [43, 44], adsorbed sulfate $\left(\mathrm{SO}_{4}^{2-}\right)$ interacts strongly with the water molecules and forms a stable mixed water-sulfate layer on $\mathrm{Au}$ and $\mathrm{Pt}$ electrodes. The stable anionic species significantly contribute to the interface dipole. A mixed water- $\mathrm{OH}^{-}$layer configuration at high electrode potential [45] has been found by Zhu et al. [42]. The solvating water structures become strongly modified at the interface, and again the stable anionic species significantly contribute to the interface dipole.

The competition between water molecules and ion species in the solvation layer can be realized by a constraint of the molecule density within the semiclassical method [33]. In the semiclassical model, the competing behavior between water molecules and ions is determined by the respective electrochemical potentials, which are derived from the grand potential of the EDL. The competition is codetermined by electrostatic, hard-sphere, and electronic interactions.

Although DFT can reproduce the interface structure with high reliability, the models must be adequately prepared for the particular electrochemical environments. When an atomic configuration does not match the considered condition and fails to reproduce the experimental situation, the results might be irrelevant for the assumed environment. For 
example, within the AIMD simulations, the relation between the interface dipole and the adsorbate-induced change on the water/electrode interaction seems opposite to the potential change. A more negatively polarized electrode requires a higher concentration of directly adsorbed water molecules blocked on the hydrogen-covered electrode. The charge in the Helmholtz layer created by the water molecules strongly bound to the electrode depends on the coverage of the corresponding water species. A higher $\mathrm{H}$ coverage causes an increase of the work function [10], which does not match the supposed electrochemical condition, i.e., $\mathrm{H}$ coverage at a low electrode potential. Adequate modeling requires simultaneous consideration of the ion distribution in the electrolyte and adsorbates on the electrode for a condition [38]. It has to be realized that AIMD simulations, due to their high numerical demand, are not the right method to yield information about the optimal adsorbate coverage and ion distribution together. Here calculations based on the semiclassical method can provide valuable information that then enters the choice of the initial configurations entering the AIMD simulations.

\section{CONCLUSION}

We have sketched the recent progress in the theoretical and numerical determination of EDL structures at electrode/electrolyte interfaces from AIMD and semiclassical approaches. Despite this recent progress, the research field requires further progress. In AIMD simulations, a proper setup of atomic configurations which can represent the desired electrochemical environments is essential. Because of the high numerical demand of AIMD simulations, the design of their initial configurations should be based on preceding grand-canonical considerations. Thus, an important role of semiclassical approaches might be to provide valuable input for AIMD simulations. Still, the semiclassical model can also be further improved with respect to a better description of the electronic structures of the electrode, a more reliable representation of ion/molecule bonds, a beyond-mean-field treatment of the electrolyte, and the coupling with micro-kinetic models. These systematic improvements should be closely based on quantum chemical arguments, including input from AIMD simulations. 


\section{DECLARE OF INTEREST}

Declare of interest: none.

\section{ACKNOWLEDGEMENT}

This research has been supported by the German Research Foundation (DFG) through contract GR 1503/39-1, the Alexander von Humboldt Foundation, and National Natural Science Foundation of China under the grant number of 21802170. Computer time provided

by the bwHPC initiative and the bwHPC-C5 project funded by the Baden-Württemberg government (MWK) and the German Research Foundation (DFG) through grant number INST 40/575-1 FUGG (JUSTUS 2 cluster) is gratefully acknowledged. This work contributes to the research performed at CELEST (Center for Electrochemical Energy Storage Ulm-Karlsruhe).

\section{REFERENCES}

- Paper of special interest

.. Paper of outstanding interest

[1] W. Schmickler, Chem. Rev. 96, 3177 (1996).

[2] A. Groß and S. Sakong, Curr. Opin. Electrochem. 14, 1 (2019).

[3] J. Le, M. Iannuzzi, A. Cuesta, and J. Cheng, Phys. Rev. Lett. 119, 016801 (2017).

[4] S. Sakong and A. Groß, J. Chem. Phys. 149, 084705 (2018).

[5] J. Le, Q. Fan, L. Perez-Martinez, A. Cuesta, and J. Cheng, Phys. Chem. Chem. Phys. 20, 11554 (2018).

[6] J.-B. Le and J. Cheng, Curr. Opin. Electrochem. 19, 129 (2020).

[7] J. Huang, P. Li, and S. Chen, Phys. Rev. B 101, 125422 (2020).

[8] J. Huang, S. Chen, and M. Eikerling, J. Chem. Theory Comput. 17, 2417 (2021).

.. This paper presents a semiclassical framework to address the grand potential of the EDL by 
combining an orbital-free quantum mechanical method and classical density-potential functional theory. The method treats the whole EDL on the same footing.

[9] J. Huang, Electrochim. Acta 389, 138720 (2021).

[10] P. Li, J. Huang, Y. Hu, and S. Chen, J. Phys. Chem. C 125, 3972 (2021).

.. This AIMD study identifies the origin of the interface dipole at the water $/ \mathrm{Pt}(111)$ interface and connects to a specific water configuration. Furthermore, it addresses the influence of $\mathrm{H}$ coverage on the dipole strength.

[11] S. Sakong, K. Forster-Tonigold, and A. Groß, J. Chem. Phys. 144, 194701 (2016).

[12] J. Le, A. Cuesta, and J. Cheng, J. Electroanal. Chem. 819, 87 (2018).

[13] J. Lan, V. V. Rybkin, and M. Iannuzzi, J. Phys. Chem. Lett. 11, 3724 (2020).

- This AIMD study discusses the proton activity at the water $/ \mathrm{Pt}(111)$ interface also regarding the nuclear quantum effects.

[14] K. Letchworth-Weaver and T. A. Arias, Phys. Rev. B 86, 075140 (2012).

[15] R. Sundararaman, W. A. Goddard, and T. A. Arias, J. Chem. Phys. 146, 114104 (2017).

[16] Y. A. Wang and E. A. Carter, Orbital-free Kinetic-energy Density Functional Theory (Springer, 2002), chap. 5, pp. 117-184, ISBN 978-0-306-46949-7.

[17] V. Gavini, K. Bhattacharya, and M. Ortiz, J. Mech. Phys. Solids 55, 697 (2007).

[18] S. Sakong and A. Groß, Phys. Chem. Chem. Phys. 22, 10431 (2020).

[19] D. Mahlberg, S. Sakong, K. Forster-Tonigold, and A. Groß, J. Chem. Theory Comput. 15, 3250 (2019).

[20] S. Trasatti, Electrochim. Acta 36, 1659 (1991).

[21] E. Skúlason, G. S. Karlberg, J. Rossmeisl, T. Bligaard, J. Greeley, H. Jónsson, and J. K. Nørskov, Phys. Chem. Chem. Phys. 9, 3241 (2007).

[22] J.-B. Le, Q.-Y. Fan, J.-Q. Li, and J. Cheng, Sci. Adv. 6, eabb1219 (2020).

[23] J.-B. Le and J. Cheng, Curr. Opin. Electrochem. 27, 100693 (2021).

[24] M. Otani and O. Sugino, Phys. Rev. B 73, 115407 (2006).

[25] M. M. Melander, M. J. Kuisma, T. E. K. Christensen, and K. Honkala, J. Chem. Phys. 150, 041706 (2019).

[26] K. Mathew, V. S. C. Kolluru, S. Mula, S. N. Steinmann, and R. G. Hennig, J. Chem. Phys. 151, 234101 (2019).

[27] S. Schnur and A. Groß, New J. Phys. 11, 125003 (2009). 
[28] N. G. Hörmann, Z. Guo, F. Ambrosio, O. Andreussi, A. Pasquarello, and N. Marzari, Npj Comput. Mater. 5, 100 (2019).

[29] G. Bramley, M.-T. Nguyen, V.-A. Glezakou, R. Rousseau, and C.-K. Skylaris, J. Chem. Theory Comput. 16, 2703 (2020).

[30] J. Liu and J. Huang, J. Electroanal. Chem. 846, 113136 (2019).

[31] J.-L. Fattebert and F. Gygi, J. Comput. Chem. 23, 662 (2002).

[32] K. Mathew, R. Sundararaman, K. Letchworth-Weaver, T. A. Arias, and R. G. Hennig, J. Chem. Phys. 140, 084106 (2014).

[33] E. Gongadze and A. Iglič, Bioelectrochemistry 87, 199 (2012).

[34] J. Haruyama, T. Ikeshoji, and M. Otani, Phys. Rev. Materials 2, 095801 (2018).

[35] R. Tesch, P. M. Kowalski, and M. H. Eikerling, J. Phys.: Condens. Matter 33, 444004 (2021).

[36] M. H. Hansen, A. Nilsson, and J. Rossmeisl, Phys. Chem. Chem. Phys. 19, 23505 (2017).

[37] R. Kronberg and K. Laasonen, J. Phys. Chem. C 124, 13706 (2020).

[38] J.-B. Le, A. Chen, L. Li, J.-F. Xiong, J. Lan, Y.-P. Liu, M. Iannuzzi, and J. Cheng, JACS Au 1, 569 (2021).

- This paper addresses tailoring the surface coverage and ions in the electrolyte simultaneously to match an electrochemical condition.

[39] S. Surendralal, M. Todorova, and J. Neugebauer, Phys. Rev. Lett. 126, 166802 (2021).

[40] H. H. Kristoffersen, T. Vegge, and H. A. Hansen, Chem. Sci. 9, 6912 (2018).

[41] H. H. Kristoffersen, K. Chan, T. Vegge, and H. A. Hansen, Chem. Commun. 56, 427 (2020).

[42] J.-X. Zhu, J.-B. Le, M. T. M. Koper, K. Doblhoff-Dier, and J. Cheng, J. Phys. Chem. C 125, 21571 (2021).

[43] F. Gossenberger, F. Juarez, and A. Groß, Front. Chem. 8, 634 (2020).

[44] Y. Fang, S.-Y. Ding, M. Zhang, S. N. Steinmann, R. Hu, B.-W. Mao, J. M. Feliu, and Z.-Q. Tian, J. Am. Chem. Soc. 142, 9439 (2020).

[45] S. Sakong, D. Mahlberg, T. Roman, M. Li, M. Pandey, and A. Groß, J. Phys. Chem. C 124, $27604(2020)$. 